\title{
Greater occipital nerve block with local anesthetics and corticosteroids in treatment-resistant chronic migraine
}

\author{
Vlasta Vukovic Cvetkovic ${ }^{1}$ (D), Roberto De Icco ${ }^{2,3}$ (D), Thien Phu Do ${ }^{1}$ (D) Lanfranco Pellesi' ${ }^{1}$ (D), \\ Messoud Ashina1 (iD, Jakob Møller Hansen ${ }^{1,4}$ (D)
}

\author{
1Danish Headache Center and Department of Neurology, Rigshospitalet Glostrup, Copenhagen, Denmark. \\ ${ }^{2}$ Headache Science Centre, IRCCS Mondino Foundation, Pavia, Italy. \\ ${ }^{3}$ Dept. of Brain and Behavioral Sciences, University of Pavia, Pavia, Italy. \\ ${ }^{4}$ Danish Headache Knowledge Center, Rigshospitalet Glostrup, Valdemar Hansens Vej, Copenhagen, Denmark.
}

\section{$凶$}

Jakob Møller Hansen, MSc, MD, PhD

Danish Headache Knowledge Center, Rigshospitalet Glostrup, Valdemar Hansens Vej, Copenhagen, Denmark.

jakob.moeller.hansen.01@ regionh.dk

Edited by: Marcelo Moraes Valença

Keywords:

Peripheral nerve block

Headache

Pain

Preventive treatment

\begin{abstract}
Objective

We investigated the efficacy and tolerability of greater occipital nerve block with lidocaine plus betamethasone in adults with chronic migraine in whom two or more previous preventive treatments were unsuccessful.

Methods

Ten participants were enrolled in a 24-weeks, randomized, double-blind, placebo-controlled, crossover trial conducted at a single tertiary headache center in Copenhagen (Denmark). After a 4-week run-in period, participants underwent treatment with bilateral greater occipital nerve block with lidocaine plus betamethasone $(\mathrm{GONb})$ or lidocaine plus saline (placebo) with a 4-week interval wash-out phase between the 8-week crossover periods. The primary aim was to compare the number of migraine days during crossover periods after $\mathrm{GONb}$ or placebo. This trial is registered at ClinicalTrials.gov (NCT02686983).

Results

This study was stopped before achieving the a priori sample size, due to a slow enrollment. Ten participants were recruited, completed the study and were included in the analyses. At the baseline, the mean number of monthly migraine days was 22.9 (range, 14-30). No difference between $\mathrm{GONb}$ and placebo on the reduction of monthly migraine days was observed ( $p=$ $0.147 ; 95 \% \mathrm{Cl}$ between 0.6 and 3.7 days). Adverse events were recorded in two patients after $\mathrm{GONb}$, compared with three patients after placebo.

\section{Conclusions}

GONb is not beneficial in patients with difficult-to-treat chronic migraine.
\end{abstract}




\section{Introduction}

$M$ igraine is a prevalent and disabling disorder charac terized by moderate to severe, throbbing headache and associated symptoms, including nausea/vomiting and hypersensitivity to light and sounds. ${ }^{1,2}$ Its chronic form (CM) is defined by the presence of at least fifteen headache days per month, of which at least eight days with the features of migraine. ' In patients with $\mathrm{CM}$, a preventive treatment is recommended. Pharmacological therapies such as antidepressants, antiepileptics, antihypertensives, botulinum toxin type $\mathrm{A}$ and monoclonal antibodies targeting the calcitonin gene-related peptide (CGRP) pathway are currently prescribed, but a variable clinical response between patients makes management challenging. ${ }^{3,4}$

Greater occipital nerve block is a peripheral nerve block technique used for the treatment of headache disorders ${ }^{5}$ with positive reports in occipital neuralgia, cluster headache, cervicogenic headache and migraine. ${ }^{6-8}$ The mechanism of action has been suggested to involve peripheral modulation of the nociceptive transmission in the caudal trigeminal nucleus and upper cervical segments. 9,10 Greater occipital nerve block performed with local anesthetics (lidocaine or bupivacaine) was beneficial in several studies for migraine prophylaxis. ${ }^{711}$ However, the addition of corticosteroids is still controversial due to scarce and heterogeneous studies. ${ }^{11-14}$

In this study, we investigated the efficacy and tolerability of greater occipital nerve block performed with lidocaine plus betamethasone (GONb) or lidocaine plus saline (placebo) in patients with $C M$ in whom two or more previous preventative treatments were unsuccessful.

\section{Methods}

The study protocol was reviewed by the Regional Health Research Ethics Committee of the Capital Region of Denmark ( $\mathrm{H}-16020715)$ and the Danish Medicines Agency (2016-000676-15). Every enrolled patient has given written informed consent to participate in the study. This trial is registered at ClinicalTrials.gov (NCT02686983).

\section{Study Participants}

Eligible patients were aged $18-75$ years, weight $<100 \mathrm{~kg}$, with a history of chronic migraine according to the International Classification of Headache Disorders' ${ }^{1}$, for at least 6 months prior to enrollment. Eligible participants also had previous treatment failure (efficacy, tolerability or both) with two or more pharmacological classes of the following migraine preventive medications: tricyclic antidepressants, antiepileptics, antihypertensives, and botulinum toxin type $\mathrm{A}$.

Individuals were excluded from the study if they have had a lifetime diagnosis of any other primary headache disorder according to the International Classification of Headache Disorders'; were pregnant or nursing; had a history of psychiatric disorder; had contraindications to corticosteroid therapy; history of illness of any kind that could preclude participation in the study according to the investigating physician; used a preventive migraine medication, device or procedure within five half-lives before the baseline phase or during the baseline phase.

\section{Randomization and Masking}

The randomization was designed to ensure an equal distribution in active-placebo and placebo-active treatment groups. Each patient was assigned a trial number corresponding to a hidden treatment order, randomized into blocks of three people. Each treatment phase included a box of a masked prefabricated medicine to ensure the doubleblinded design. Thus, the blinded design was ensured by concealed allocation, and masking of treatment.

Randomization code, medicine and placebo were prepared by the Central Pharmacy of the Capital Region of Denmark. At the end of each period, a blinding check was made by asking if the participant believed they had received active or placebo treatment.

\section{Study Design and Procedures}

The current study was a 24-week, randomized, double-blind, placebo-controlled crossover trial conducted at a single tertiary headache center in Copenhagen (Denmark). The study included a screening visit, a baseline assessment period (4 weeks) and two double-blind periods (8 weeks each), with a washout-phase in between (4 weeks) (Figure 1). During screening, all participants underwent a physical examination and eligibility assessments. During the baseline assessment period, participants recorded headache, migraine symptoms and use of rescue medications.

Randomized participants received treatment on day 1 of the first 8-week double-blind period. Before entering the second double-blind period, participants completed the 4-week washout-phase and received the second treatment. The 
active treatment consisted of bilateral greater occipital nerve block injections with $0.1 \mathrm{ml}$ of $4 \%$ lidocaine plus $7 \mathrm{mg}$ of betamethasone $(\mathrm{GONb})$, while placebo consisted of bilateral injections of $0.1 \mathrm{ml}$ of $4 \%$ lidocaine plus saline. Patient was placed in a sitting position, with the head slightly bent to the side. The injection area was identified $2.0-2.5 \mathrm{~cm}$ lateral and $2.0-2.5 \mathrm{~cm}$ inferior to the protuberantia occipitalis. The injection site was cleansed with alcohol and the injection was performed in the most tender area to palpation, with a 23-25 $G$ needle over the identified area, bilaterally.

\section{Figures' captions}

(NRS) from 0 to 10. Safety was also assessed by observed or reported adverse events and vital signs assessments.

\section{Statistical Analysis}

Sample size was calculated with the following parameters: mean difference of 5 migraine days between $\mathrm{GONb}$ and placebo; standard deviation of $10 \%$ per group; level of significance of $5 \%$ (two-sided); power of $80 \%$. The suggested sample size was of 34 patients, 17 of whom allocated to each treatment group.

Quantitative data are presented as "mean (range)", and

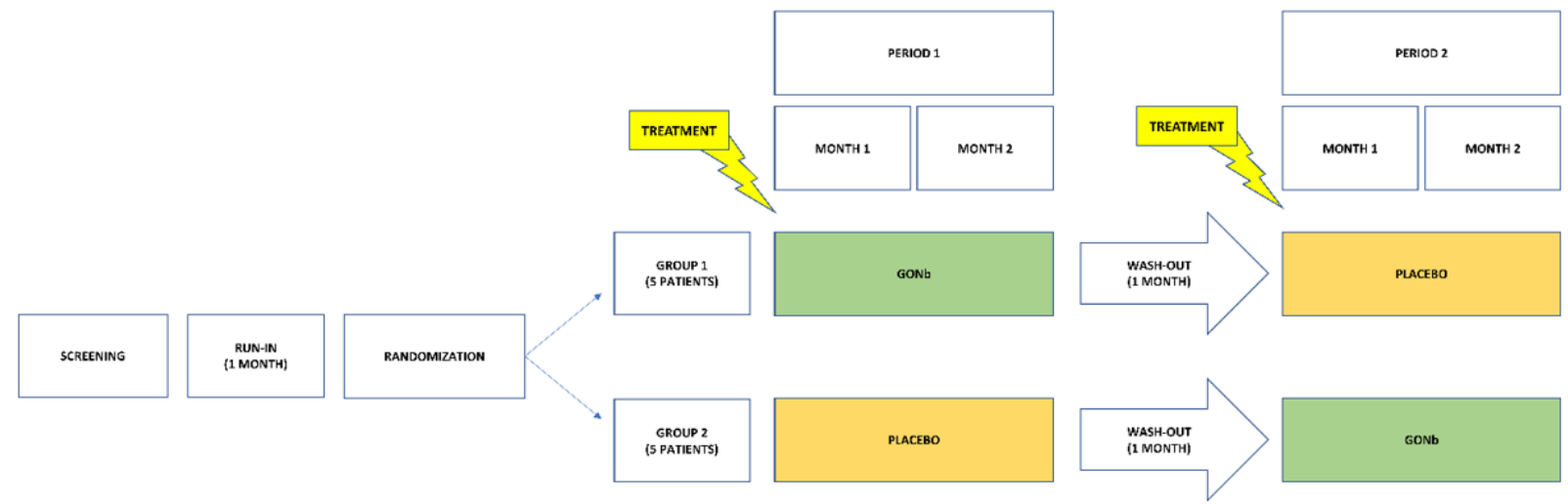

Figure 1. Flow-chart of the study procedures. GONb: greater occipital nerve block with lidocaine plus betamethasone. Placebo: greater occipital nerve block with lidocaine plus saline. Group 1 included patients randomized to GONb as first treatment. Group 2 included patients randomized to placebo as first treatment.

During the double-blind periods, participants recorded daily data in their headache diary. Moreover, each participant was contacted by phone four times during the trial: midway through each treatment period and at the end of each treatment period. At the end of each treatment period, patients were asked to rate their satisfaction with the treatment by mean of a 1 (very dissatisfied) to 5 (very satisfied) points Likert scale.

\section{Outcomes}

The primary endpoint was comparing the number of monthly migraine days during periods after active treatment (GONb) with periods after placebo. A migraine day was defined as a calendar day with at least four consecutive hours of a headache fulfilling the diagnostic criteria for "migraine without aura" or "probable migraine" according to the International Classification', or a headache of any duration successfully treated with an acute migraine-specific medication.

Secondary endpoints were the change in the number of headache days per month; change in the number of days using acute migraine-specific medications per month; change of headache intensity measured by a numerical rating scale categorical data as "absolute number (percentage)". Baseline characteristics between groups were compared with MannWhitney $\mathrm{U}$ test, while comparisons for categorical variables were performed with Pearson $x^{2}$ test or Fisher exact test. Change in primary outcome (monthly migraine days) as well as in secondary outcomes (monthly headache days, monthly days of acute migraine medications, and headache intensity) were analyzed with linear mixed models using the above described outcomes as dependent variable. As fixed-effect factors we assumed: factor "treatment" (2 levels: GONb vs. placebo), and factor "period" (2 levels: first double-blind period vs. second double-blind period). Patient ID was used as random-effect factor. Comparisons of adverse events and blinding check were performed with McNemar's test. Patients satisfaction was evaluated with Mann-Whitney $U$ test. The level of significance was set at $a=0.050$ for all tests. The Statistical Package for the Social Sciences (SPSS), version 21.0 for Windows, was used for all the computations.

\section{Results}

\section{Baseline Characteristics of the Study Population}


Between April 2017 and August 2019, 80 patients were screened for eligibility and ten were enrolled in the study (Figure 1). This study was stopped before achieving the a priori sample size, due to a slow enrollment. Ten patients were included; five were randomly assigned to active-placebo group (Group 1) and five to placebo-active group (Group 2). Both groups were balanced in terms of baseline demographic and disease characteristics (Table 1). We did not find a carry-over effect for all primary and secondary outcomes, no interaction between fixed-effect factors occurred. Moreover, the period effect was not different for all outcomes (Table 2).

Table 1. Clinical and demographic features of the study population

\begin{tabular}{|c|c|c|c|c|}
\hline & & Study population & Group 1 & Group 2 \\
\hline \multicolumn{2}{|l|}{$\mathrm{N}$} & 10 & 5 & 5 \\
\hline \multicolumn{2}{|l|}{ Age } & $38.7(20-57)$ & $46.2(36-57)$ & $\begin{array}{l}31.2(17.0 . \\
45.4)\end{array}$ \\
\hline \multicolumn{2}{|l|}{ Female sex } & $8(80.0 \%)$ & $4(80.0 \%)$ & $4(80.0 \%)$ \\
\hline \multicolumn{2}{|l|}{ Height $(\mathrm{cm})$} & $174.1(164-186)$ & $\begin{array}{l}175.6(165 \\
183)\end{array}$ & $\begin{array}{l}172.6(164- \\
186)\end{array}$ \\
\hline \multicolumn{2}{|l|}{ Weight (Kg) } & $78.6(61-95)$ & $81.2(61-95)$ & $76.0(65-90)$ \\
\hline \multicolumn{2}{|c|}{ Systolic blood pressure $(\mathrm{mmHg})$} & $126.5(105-161)$ & $\begin{array}{l}133.0(106 \\
161)\end{array}$ & $\begin{array}{l}120.0(105- \\
133)\end{array}$ \\
\hline \multicolumn{2}{|c|}{ Diastolic blood pressure $(\mathrm{mmHg})$} & $83.6(67-94)$ & $88.0(78-94)$ & $79.2(67-87)$ \\
\hline \multicolumn{2}{|l|}{ Heart rate (bpm) } & $76.7(66-97)$ & $75.6(69-84)$ & $77.8(66-97)$ \\
\hline \multicolumn{2}{|c|}{ Migraine days per month } & $22.9(14-30)$ & $21.2(14.28)$ & $24.6(15-30)$ \\
\hline \multicolumn{2}{|c|}{ Headache days per month } & $26.4(16-30)$ & $25.6(16-30)$ & $27.2(16-30)$ \\
\hline \multicolumn{2}{|c|}{ Days of acute drug intake per month } & $7.1(0-12)$ & $8.6(6-11)$ & $5.6(0-12)$ \\
\hline \multicolumn{2}{|c|}{ Doses of acute drugs per month } & $9.9(0-26)$ & $11.4(6-15)$ & $8.4(0-26)$ \\
\hline \multicolumn{2}{|c|}{ Headache intensity (NRS) } & $5.9(3.7-8.1)$ & $5.4(3.9-7.2)$ & $6.5(3.7-8.1)$ \\
\hline \multicolumn{2}{|c|}{ Medication overuse } & $3(30.0 \%)$ & $1(20.0 \%)$ & $2(40.0 \%)$ \\
\hline \multirow{7}{*}{$\begin{array}{l}\text { Previous } \\
\text { preven tive } \\
\text { treatment } \\
\text { failures }\end{array}$} & NSAID & $1(10.0 \%)$ & $0(0.0 \%)$ & $1(20.0 \%)$ \\
\hline & Triptan & $5(50.0 \%)$ & $4(80.0 \%)$ & $1(20.0 \%)$ \\
\hline & Opioid & $1(10.0 \%)$ & $0(0.0 \%)$ & $1(20.0 \%)$ \\
\hline & Multiple & $3(30.0 \%)$ & $1(20.0 \%)$ & $2(40.0 \%)$ \\
\hline & 2 & $1(10.0 \%)$ & $1(20.0 \%)$ & $0(0.0 \%)$ \\
\hline & 3 & $1(10.0 \%)$ & $1(20.0 \%)$ & $0(0.0 \%)$ \\
\hline & $\geq 4$ & $8(80.0 \%)$ & $3(60.0 \%)$ & $5(100.0 \%$ \\
\hline
\end{tabular}

NRS: numerical rating scale. NSAID: nonsteroidal anti-inflammatory drug. GONb: greater occipital nerve block with lidocaine plus betamethasone. Placebo: greater occipital nerve block with lidocaine plus saline. Group 1 included patients randomized to GONb as first treatment. Group 2 included patients randomized to placebo as first treatment. Data are presented as "mean (range)", or "absolute number (percentage)"

\section{Migraine and Headache Days}

Patients from the Group 1 reported a mean of 23.1 (16.029.5) monthly migraine days after $\mathrm{GONb}$, and a mean of 22.0 (16.0-29.5) monthly migraine days after placebo. Patients from the Group 2 reported a mean of 24.8 (15.030.0 ) monthly migraine days after $\mathrm{GONb}$, and a mean of 22.8 (1 1.0-30.0) monthly migraine days after placebo. There was no difference between $\mathrm{GONb}$ compared to placebo (factor "treatment": $p=0.147$, effect size GONb vs. placebo: 1.5 days, $95 \% \mathrm{Cl}$ between -0.6 and 3.7 days) (Table 2 and Figure 2). Patients from the Group 1 reported a mean of 26.0 (16.0-30.0) monthly headache days after $\mathrm{GONb}$, and a mean of 24.2 (16.0-29.5) monthly headache days after placebo. Patients from the Group 2 displayed a mean of 25.4 (15.0-30.0) monthly headache days after $\mathrm{GONb}$, and a mean of 25.5 (13.0-30.0) monthly headache days after placebo. There was no difference between $\mathrm{GONb}$ compared to placebo (factor "treatment": $p=0.164$, effect size GONb vs. placebo: 0.8 days, $95 \% \mathrm{Cl}$ between -0.4 and 2.1 days) (Table 2 and Figure 3).

\section{Acute Migraine-Specific Medications}

Patients from the Group 1 reported a mean of 9.7 (9.0-10.5) days of acute migraine-specific medications during $\mathrm{GONb}$, and a mean of 9.0 (7.0-1 1.5) days during placebo. Patients from the Group 2 displayed a mean of 6.3 (0.0-12.5) days of acute migraine-specific medications during $\mathrm{GONb}$, and a mean of $4.5(0.0-10.0)$ days during placebo. There was no difference between GONb compared to placebo (factor "treatment": $p=0.076$, effect size GONb vs. placebo: 1.3 medications, $95 \% \mathrm{Cl}$ between -0.1 and 2.6 medications) (Table 2 and Figure 2).

\section{Headache Intensity}

Patients in the Group 1 reported a mean NRS of 5.6 (range: 4.9-7.2) after GONb, and a mean NRS of 5.7 (range: 4.97.7) after placebo. Patients in the Group 2 reported a mean NRS of 7.1 (range: 4.4-8.9) after the GONb, and a mean NRS of 6.8 (range: 4.5-7.9) after placebo. There was no difference between GONb compared to placebo (factor "treatment": $p=0.503$, effect size GONb vs. placebo: 0.1, 95\% $\mathrm{Cl}$ between -0.2 and 0.4 ) (Table 2 and Figure 2).

\section{Blinding Check and Patient Satisfaction}

Four out of 10 patients (40\%) after $\mathrm{GONb}$, and 2 out of 10 patients $(20 \%)$ after placebo declared they received active treatment $(p=0.628)$. The average score for the satisfaction scale was 2.6 (1-4) after GONb, and 2.2 (1-4) after placebo $(p=0.481)$.

\section{Safety}

All patients from both groups completed the study. Overall, the procedure was well tolerated either with GONb or placebo. Two patients reported at least one adverse event (AE) after $G O N b$, while three patients reported at least one $A E$ after 
Table 2. Clinical findings during the cross-over trial

\begin{tabular}{|c|c|c|c|c|c|c|c|}
\hline & \multirow{2}{*}{ Baseline } & \multicolumn{3}{|c|}{ First period } & \multicolumn{3}{|c|}{ Second period } \\
\hline & & Month 1 & Month 2 & Average & Month 1 & Month 2 & Average \\
\hline \multicolumn{8}{|c|}{ Monthly Migraine Days } \\
\hline Group 1 & $21.2(14-28)$ & $23.4(15-30)$ & $22.8(17-29)$ & $23.1(16.0-29.5)$ & $23.4(16-30)$ & $20.4(16-29)$ & $22.0(16.0-29.5)$ \\
\hline Group 2 & $24.6(15-30)$ & $21.4(11-30)$ & $24.2(10-30)$ & $22.8(11.0-30.0)$ & $23.8(16-30)$ & $25.8(14-30)$ & $24.8(15.0-30.0)$ \\
\hline \multicolumn{8}{|c|}{ Monthly Headache Days } \\
\hline Group 1 & $25.6(16-30)$ & $26.2(15-30)$ & $25.8(17-30)$ & $26.0(16.0-30.0)$ & $24.6(16-30)$ & $23.8(16-29)$ & $24.2(16.0-29.5)$ \\
\hline Group 2 & $27.2(16-30)$ & $25.0(13-30)$ & $26.0(13-30)$ & $25.5(13.0-30.0)$ & $24.6(16-30)$ & $26.2(14-30)$ & $25.4(15.0-30.0)$ \\
\hline \multicolumn{8}{|c|}{ Acute Migraine-Specific Medications } \\
\hline Group 1 & $8.6(6-11)$ & $10.6(9-14)$ & $8.8(5-11)$ & $9.7(9.0-10.5)$ & $9.2(8-10)$ & $8.8(0-14)$ & $9.0(7.0-11.5)$ \\
\hline Group 2 & $5.6(0-12)$ & $4.0(0-9)$ & $5.0(0-11)$ & $4.5(0.0-10.0)$ & $6.2(0-11)$ & $6.4(5-13)$ & $6.3(0.0-12.5)$ \\
\hline \multicolumn{8}{|c|}{ Headache intensity } \\
\hline Group 1 & $5.4(3.9-7.2)$ & $5.9(5.1-7.5)$ & $5.4(3.8-7.0)$ & $5.6(4.9-7.2)$ & $5.8(4.9-8.1)$ & $5.6(4.9-7.2)$ & $5.7(4.9-7.7)$ \\
\hline Group 2 & $6.5(3.7-8.1)$ & $6.7(4.1-7.9)$ & $6.8(4.8-8.0)$ & $6.8(4.5-7.9)$ & $6.9(4.2-9.0)$ & $7.1(4.5-8.8)$ & $7.1(4.4-8.9)$ \\
\hline
\end{tabular}

Headache intensity was measured with a numerical rating scale (NRS) from 0 to 10 . Group 1 included patients randomized to GONb as first treatment. Group 2 included patients randomized to placebo as first treatment. GONb: greater occipital nerve block with lidocaine plus betamethasone. Placebo: greater occipital nerve block with lidocaine plus saline. Grey shades: periods after active treatment. Data are presented as "mean (range)".
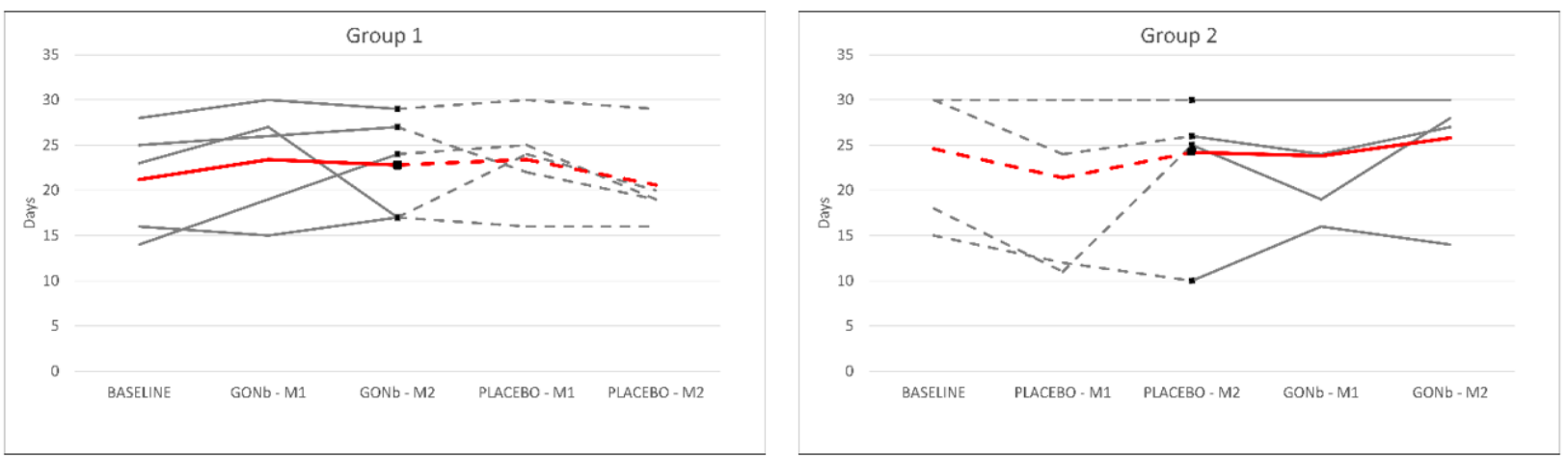

Figure 2. Change in migraine days per month. Panel on the left describes changes in Group 1. Panel on the right describes changes in Group 2. Grey lines: estimates of individual patients. Red lines: mean values. Continuous lines: study period after GONb. Dashed lines: study period after placebo. M1: first month of the period; M2: second month of the period. GONb: greater occipital nerve block with lidocaine plus betamethasone. Placebo: greater occipital nerve block with lidocaine plus saline. 

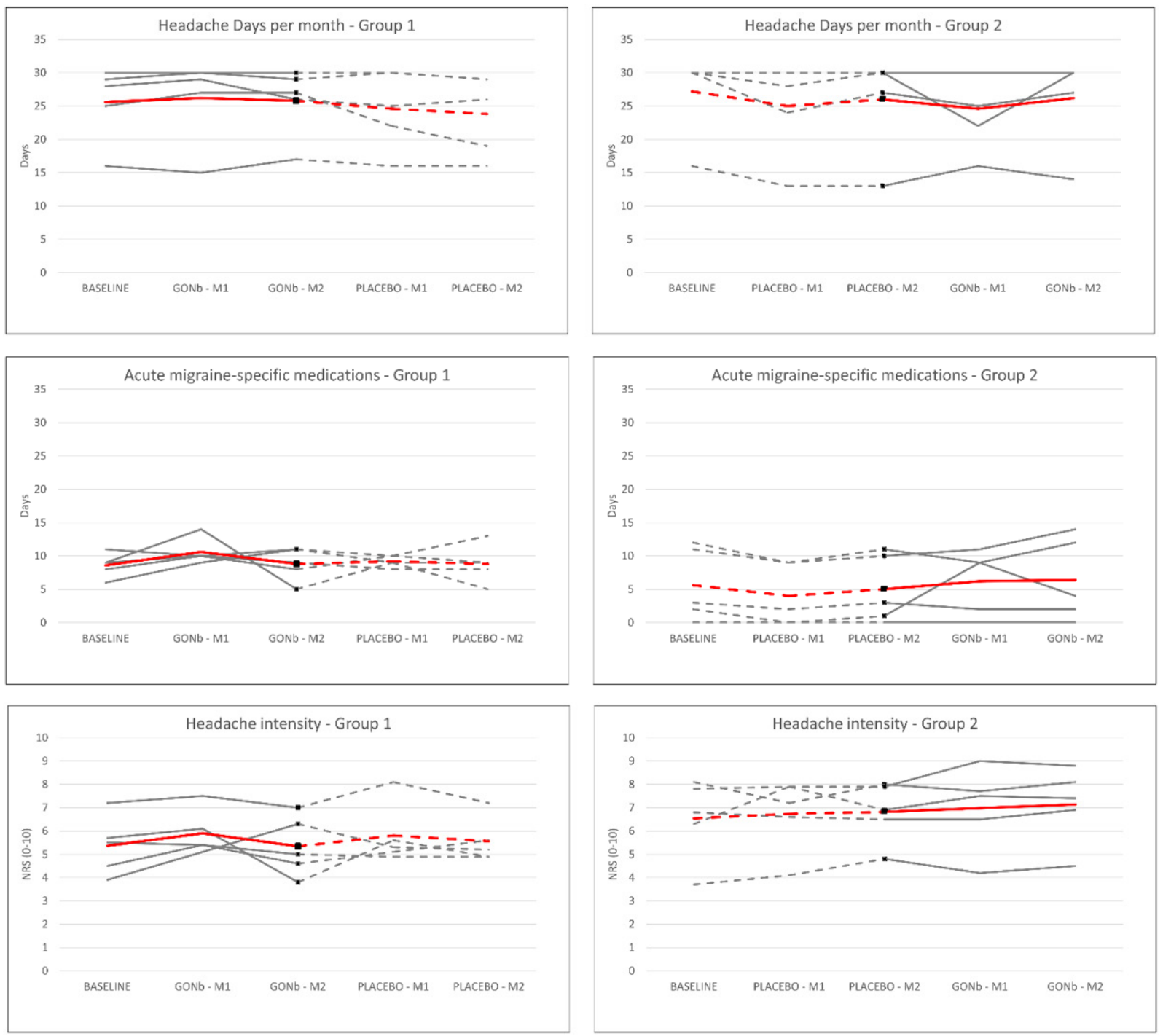

Figure 3. Changes of secondary outcomes. Panels on the left describe changes in Group 1. Panels on the right describe changes in Group 2. Grey lines: estimates of individual patients. Red lines: mean values. Continuous lines: study period after GONb. Dashed lines: study period after placebo. M1: first month of the period; M2: second month of the period. NRS: numerical rating scale. GONb: greater occipital nerve block with lidocaine plus betamethasone. Placebo: greater occipital nerve block with lidocaine plus saline.

placebo $(p=1.000)$. Eight AEs were reported in total, all of treatments. There was not clinically meaningful or statistical them being transitory, treatment-related and mild in intensity. Five AEs were reported after $\mathrm{GONb}$, and three after placebo. The most frequent $\mathrm{AE}$ was muscle tension at the neck level.

\section{Discussion}

The main finding of this cross-over study is that GONb with corticosteroids was not beneficial in CM-patients with several prior treatment failures. To our knowledge, this is the first cross-over trial investigating the preventive effect of $\mathrm{GONb}$ technique with lidocaine plus betamethasone in patients with $C M$, with a history of multiple unsuccessful pharmacological difference between $\mathrm{GONb}$ and placebo for either primary or secondary efficacy endpoints, including migraine days, headache days, acute medication days, and headache intensity. Adverse events reported after GONb were not different to those reported after placebo.

$\mathrm{GONb}$ with corticosteroids may be considered a further therapeutic opportunity for chronic headaches refractory to conventional preventive therapies. ${ }^{14,15} \mathrm{~A}$ recent meta-analysis reported a clinical benefit of greater occipital nerve block with local anesthetics for the preventive treatment of chronic migraine. ${ }^{16}$ However, the results of the meta-analysis are 
affected by several methodological limitations, including pooling together different study designs. In our study, the population was comprised of patients with $\mathrm{CM}$ with at least two previous unsuccessful pharmacological preventive treatments, who are typically excluded in randomized clinical trials. The current findings are consistent with trials with comparable study designs. Two studies reported no difference between patients who received local anesthetics plus triamcinolone compared to patients who received only anesthetics. ${ }^{12,14}$ Also, one study found no diversity between $\mathrm{GONb}$ performed with bupivacaine plus methylprednisolone or lidocaine alone. ${ }^{13}$

\section{Strengths and Limitations}

The strength of this study includes the enrollment of a deeply characterized cohort of patients with difficult-to-treat CM and use of clinical outcomes as recommended by the International Headache Society. ${ }^{17}$ Furthermore, the cross-over design reduced variability, as patients represented their own controls. The use of blinding check revealed no masking issues during the double-blind treatment phase. The major limitation was the low number of recruited patients, which was lower than expected. The primary reason was that ongoing competitive clinical trials with the new anti-CGRP monoclonal antibodies were offered to the same patient population. At the same time, the recorded effect size for the primary outcome (GONb vs. placebo: 1.5 days, $95 \% \mathrm{Cl}$ between -0.6 and 3.7 days), was far from the clinically meaningful difference we assumed for the sample size calculation. The small sample may have limited statistical detection of significant changes in endpoints, but we emphasize that no clinical benefit in individual patients was observed. This is also reinforced by the low grade of satisfaction expressed by patients after both $\mathrm{GONb}$ and placebo. Regardless, our results should be interpreted with caution.

\section{Conclusion}

This study expands data about the effective use of greater occipital nerve block in the prevention of CM. Greater occipital nerve block with lidocaine and betamethasone has no benefit in patients with $C M$, with a history of two or more previous unsuccessful pharmacological preventive treatments.

Conflicts of Interest: WVC, RDI, TPD and LP declare no competing interests. JMH reports personal fees from Eli Lilly, Novartis and Teva. MA reports personal fees from Alder, Allergan, Amgen, Eli Lilly, Lundbeck, Novartis and Teva. MA participated in clinical trials as the principal investigator for Alder, Amgen, ElectroCore, Eli Lilly, Novartis and Teva trials. $M A$ reports receiving consulting fees and advisory boards fees from Alder, Allergan, Amgen, Eli Lilly, Lundbeck, Novartis, and
Teva, fees for serving as a principal investigator, paid to his institution, from Alder, Allergan, Amgen, Electro-Core, Eli Lilly, Lundbeck, Novartis, and Teva, and grant support, paid to his institution, from Novo Nordisk Foundation, Novartis, and Lundbeck Foundation.

Author Contributions: WV: contributed to study conception and design; contributed to data acquisition and interpretation; critically revised the manuscript before final approval.

RDI: performed statistical analysis; contributed to data interpretation; contributed to first draft of the manuscript; critically revised the manuscript before final approval.

TPD: contributed to data acquisition and interpretation; contributed to first draft of the manuscript; critically revised the manuscript before final approval.

LP: contributed to data interpretation; contributed to first draft of the manuscript; critically revised the manuscript before final approval.

MA: contributed to study conception and design; contributed to data interpretation; critically revised the manuscript before final approval.

$\mathrm{JMH}$ : contributed to study conception and design; contributed to data acquisition and interpretation; critically revised the manuscript before final approval.

All authors read and approved the final manuscript.

Vlasta Vukovic Cvetkovic

https://orcid.org/0000-0003-4963-4541

Roberto De lcco

https://orcid.org/0000-0001-9415-4948

Thien Phu Do

https://orcid.org/0000-0002-9631-0665

Lanfranco Pellesi

https://orcid.org/0000-0003-4137-5039

Messoud Ashina

https://orcid.org/0000-0003-0951-5804

Jakob Møller Hansen

https://orcid.org/0000-0002-9417-2481

\section{References}

1. The International Classification of Headache Disorders, 3rdedition (betaversion). Cephalalgia2013;33(9):629808 Doi: $10.1177 / 0333102413485658$

2. Lanteri-Minet M. Economic burden and costs of chronic migraine. Curr Pain Headache Rep 2014;18(1):385 Doi:10.1007/s1 1916-013-0385-0

3. Hepp Z, Dodick DW, Varon SF, Gillard P, Hansen $R N$ and Devine EB. Adherence to oral migrainepreventive medications among patients with chronic migraine. Cephalalgia 2015;35(6):478-488 Doi: $10.1177 / 0333102414547138$

4. Hepp Z, Dodick DW, Varon SF, Chia J, Matthew N, 
Gillard $P, \ldots$ Devine EB. Persistence and switching patterns of oral migraine prophylactic medications among patients with chronic migraine: A retrospective claims analysis. Cephalalgia 2017;37(5):470-485 Doi:10.1177/0333102416678382

5. Ashkenazi A, Blumenfeld A, Napchan U, Narouze S, Grosberg B, Nett R, . . . Lipton RB. Peripheral nerve blocks and trigger point injections in headache management - a systematic review and suggestions for future research. Headache 2010;50(6):943-952 Doi: $10.1111 /$ j.1526-4610.2010.01675.x

6. Caputi CA and Firetto V. Therapeutic blockade of greater occipital and supraorbital nerves in migraine patients. Headache 1997;37(3):174-179 Doi:10.1046/i.1526-4610.1997.3703174.x

7. Afridi SK, Shields KG, Bhola R and Goadsby PJ. Greater occipital nerve injection in primary headache syndromes--prolonged effects from a single injection. Pain 2006;122(1-2):126-129 Doi:10.1016/j. pain.2006.01.016

8. Naja ZM, El-Rajab M, Al-Tannir MA, Ziade FM and Tawfik OM. Occipital nerve blockade for cervicogenic headache: a double-blind randomized controlled clinical trial. Pain Pract 2006;6(2):89-95 Doi:10.1111/j.1533-2500.2006.00068.x

9. Busch V, Jakob W, Juergens T, Schulte-Mattler W, Kaube $\mathrm{H}$ and May $\mathrm{A}$. Functional connectivity between trigeminal and occipital nerves revealed by occipital nerve blockade and nociceptive blink reflexes. Cephalalgia 2006;26(1):50-55 Doi:10.1111/ j. 1468-2982.2005.00992.x

10. Piovesan EJ, Kowacs PA, Tatsui CE, Lange MC, Ribas LC and Werneck LC. Referred pain after painful stimulation of the greater occipital nerve in humans: evidence of convergence of cervical afferences on trigeminal nuclei. Cephalalgia 2001;21(2):107-109 Doi:10.1046/j.1468-2982.2001.00166.x
11. Inan LE, Inan N, Unal-Artık HA, Atac Cand Babaoglu G. Greater occipital nerve block in migraine prophylaxis: Narrative review. Cephalalgia 2019;39(7):908-920 Doi:10.1177/0333102418821669

12. Ashkenazi A, Matro R, Shaw JW, Abbas MA and Silberstein SD. Greater occipital nerve block using local anaesthetics alone or with triamcinolone for transformed migraine: a randomised comparative study. J Neurol Neurosurg Psychiatry 2008;79(4):415417 Doi:10.1136/innp.2007.124420

13. Dilli E, Halker R, Vargas B, Hentz J, Radam T, Rogers R and DodickD. Occipital nerve block for the short-term preventive treatment of migraine: A randomized, double-blinded, placebo-controlled study. Cephalalgia 2015;35(1 1):959968 Doi: $10.1177 / 0333102414561872$

14. Kashipazha D, Nakhostin-Mortazavi A, Mohammadianinejad SE, Bahadoram M, Zandifar S and Tarahomi S. Preventive effect of greater occipital nerve block on severity and frequency of migraine headache. Glob J Health Sci 2014;6(6):209-213 Doi: 10.5539/gihs.v6n6p209

15. Martelletti P, Giamberardino MA and Mitsikostas DD. Greater occipital nerve as target for refractory chronic headaches: from corticosteroid block to invasive neurostimulation and back. Expert Rev Neurother 2016;16(8):865-866 Doi:10.1586/1473 7175.2016 .1164599

16. Shauly O, Gould DJ, Sahai-Srivastava S and Patel KM. Greater Occipital Nerve Block for the Treatment of Chronic Migraine Headaches: A Systematic Review and Meta-Analysis. Plast Reconstr Surg 2019; 144(4):943952 Doi:10.1097/prs.0000000000006059

17. Tassorelli C, Diener HC, Dodick DW, Silberstein SD, Lipton RB, Ashina M, . . Wang SJ. Guidelines of the International Headache Society for controlled trials of preventive treatment of chronic migraine in adults. Cephalalgia 2018;38(5):815832 Doi:10.1177/0333102418758283 